\title{
Development of General-Purpose Software to Analyze the Static Thermal Characteristic of Nuclear Power Plant*
}

\author{
Yoshinobu NAKAO $^{* *}$, Eiichi KODA ${ }^{* *}$ and Toru TAKAHASHI ${ }^{* *}$ \\ ${ }^{* *}$ Central Research Institute of Electric Power Industry, \\ 2-6-1 Nagasaka, Yokosuka-shi, Kanagawa-ken, 240-0196, Japan \\ E-mail: y-nakao@criepi.denken.or.jp
}

\begin{abstract}
We have developed the general-purpose software by which static thermal characteristic of the power generation system is analyzed easily. This software has the notable features as follows.

- It has the new algorithm to solve non-linear simultaneous equations to analyze the static thermal characteristics such as heat and mass balance, efficiencies, etc. of various power generation systems.

- It has the flexibility for setting calculation conditions.

- It is able to be executed on the personal computer easily and quickly.

We ensured that it is able to construct heat and mass balance diagrams of main steam system of nuclear power plant and calculate the power output and efficiencies of the system. Furthermore, we evaluated various heat recovery measures of steam generator blowdown water and found that this software could be a useful operation aid for planning effective changes in support of power stretch.
\end{abstract}

Key words: General-Purpose Software, Static Thermal Characteristic, Heat and Mass Balance Diagram, Nuclear Power Plant

\section{Introduction}

Up until a few years ago, nuclear power plants in Japan were operated at the rated electrical output. In recent days, however, these plants are operated at the rated heat output, and, a variety of measures are being proposed for power stretch and higher thermal efficiency. From the standpoint of energy utilization effectively, it is desirable to generate more electrical output on the same heat output, it is necessary to quantitatively evaluate the influence of these measures on thermal efficiency of the power plant. Generally, to analyze the performance of power generation system, it is necessary to make calculation program for each system and it is difficult to compare the calculation results under different conditions. With some commercially available software, analysis object is limited and a degree of freedom for condition setting is low.

On the other hand, we formed the committee to study the issues facing electric power companies and the needs for plant performance management, and we have developed the general-purpose software to analyze the static thermal characteristic of various power generation system; thermal ${ }^{[1],[2]}$, geothermal ${ }^{[3]}$, fuel cell, IGCC (integrated coal gasification combined cycle) power generation system, and so on. Because we have created this entire program using the new algorithm we designed, this software has the ability to solve non-linear simultaneous equations of power generation system very quickly and the flexibility to set a variety of calculation conditions and build new system component models which reflect the latest research achievements.

In this paper, we introduce the analytical method of this software, and then the application example, 
which we calculated and evaluated various heat recovery measures of steam generator blowdown water in main steam system of a nuclear power plant.

\section{Analytical method for power plant}

The nuclear power plant system consists of a steam generator, saturated steam turbines, a moisture separator, heat exchangers, a condenser, etc. Laws of energy and mass conservation are applied to every plant component. The static characteristic analysis of the power plant system is to solve all these mathematical expressions used to describe the working fluids, which flow in or out to the individual system components. In these expressions, non-linear equations might be included. Therefore, general-purpose software that can solve a huge matrix of these non-linear simultaneous equations is necessary to analyze that of power plant system.

However, some problems remain unaddressed in such software.

- The computation time lengthens very much with increasing number of component.

- It does not have the flexibility for setting calculation conditions.

Here, in this paper, we describe the new algorithm of this software. Although it is combinatorial algorithm of various typical methods, we have gotten a Japanese patent ${ }^{[4]}$ as software which has the flexibility for setting the calculation condition and for building new system component models into the software, and the ability to calculate the static thermal characteristic of power plant faster.

\subsection{Linearization of expression of each component}

Each system component has linearized equations of total pressure; $P(=$ static pressure + dynamic pressure ), amount of mass flow; $G$ and enthalpy; $H$, which are derived by laws of energy and mass conservation. And non-linear relational expressions by which $P$ is calculated from $G, P$ from $H$, and $G$ from $H$ (see Fig. 1). Then the equation for entire system is obtained by combining these expressions of each system component. In this software, by executing the balance calculations related to $P, G$, and $H$ separately, all the equations are linearized. Instead, convergence calculation is necessary to determine suitable values of the variables of each system component.

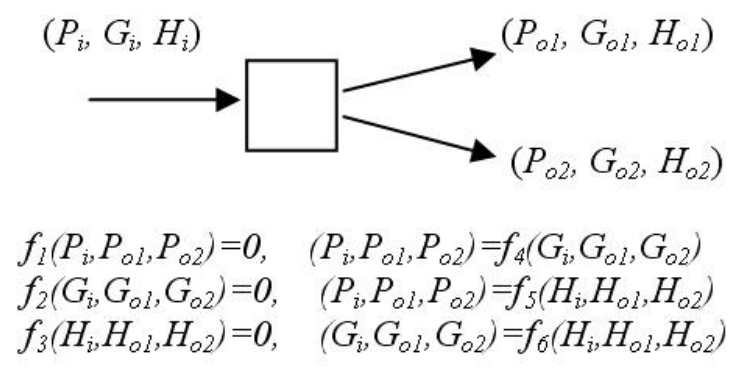

Fig. 1 Expressions for each system component

\subsection{Flexibility for setting calculation conditions}

If the following expression is embedded in a code, the calculation becomes one-way, from $P_{i n}$ to $P_{\text {out }}$.

$$
\begin{aligned}
P_{\text {out }}=P_{\text {in }} & *(1-d p) \\
& P_{\text {in }}: \text { In-stream pressure } \\
& P_{\text {out }}: \text { Out-stream pressure } \\
& d p: \text { ratio of pressure drop }
\end{aligned}
$$


On the other hand, if the following expression is embedded, it is more flexible to set the calculation conditions.

$$
(1-d p,-1)\left(P_{\text {in }}, P_{\text {out }}\right)^{T}=(0)
$$

In this case, when the value of $P_{i n}$ is set, Eq.(2) and the equation ' $P_{i n}=c$ ' make simultaneous equations as follows.

$$
\left(\begin{array}{cc}
1-d p & -1 \\
1 & 0
\end{array}\right)\left(\begin{array}{l}
P_{\text {in }} \\
P_{\text {out }}
\end{array}\right)=\left(\begin{array}{l}
0 \\
c
\end{array}\right)
$$

Similarly, if constant $c$ is set to $P_{\text {out }}$, the equation becomes following.

$$
\left(\begin{array}{cc}
1-d p & -1 \\
0 & 1
\end{array}\right)\left(\begin{array}{c}
P_{\text {in }} \\
P_{\text {out }}
\end{array}\right)=\left(\begin{array}{l}
0 \\
c
\end{array}\right)
$$

Thus, we can set $P_{\text {in }}$ or $P_{\text {out }}$ as a calculation condition. All the expressions related to $P, G$, and $H$ are given in the form of vectors of coefficients and variables, and simultaneous equation of entire system is derived by combining those expressions. Therefore, this program has the flexibility for setting the calculation conditions.

Moreover, since the program is designed using object oriented programming, if we have to build a new system component into the software, we just add a class object of the component model and code an expression like Eq.(2) in the software. Therefore, we need to incorporate the high-speed core program that permits it to arrange the expressions of each component in a matrix and solve the huge matrix.

\subsection{Calculation Method for the entire system}

The equations of each component included in the system are arranged and sorted as follows

$$
\begin{aligned}
\boldsymbol{A}_{i} \cdot \boldsymbol{x}=C_{i} & \\
& i:=1 . . n \\
& n: \text { number of variables } \\
& \boldsymbol{A}_{i}: \text { vector of coefficients } \\
& \boldsymbol{x}: \text { vector of variables } \\
& C_{i}: \text { constants }
\end{aligned}
$$

Then the equation for entire system is given by

$$
\mathbf{A} \cdot \boldsymbol{x}=\boldsymbol{c}
$$
A: matrix of coefficients
c: vector of constants

The solution can be obtained by solving this Eq.(6). However, there remained the following problems.

- The matrix may become very huge

- There is no guarantee that the number of equations is corresponding to the number of variables. Moreover, it is not guaranteed that the equations are all independent.

So, in order to make this technique available to various systems under various calculation conditions, the factorization method of the matrix and the checking method of the equations are necessary. 


\section{Reducing the size of matrix}

To reduce the size of matrix, the balance calculations related to $P, G$, and $H$ are executed respectively. For example, in the calculation of the power plant system, the number of variables associated with the mass flow rate becomes overwhelming. On the other hand, there are few components in which the mass flow rate or composition of working fluid changes. The size of the matrix is reduced significantly by omitting the expressions which have an entrance value equal to that of the exit.

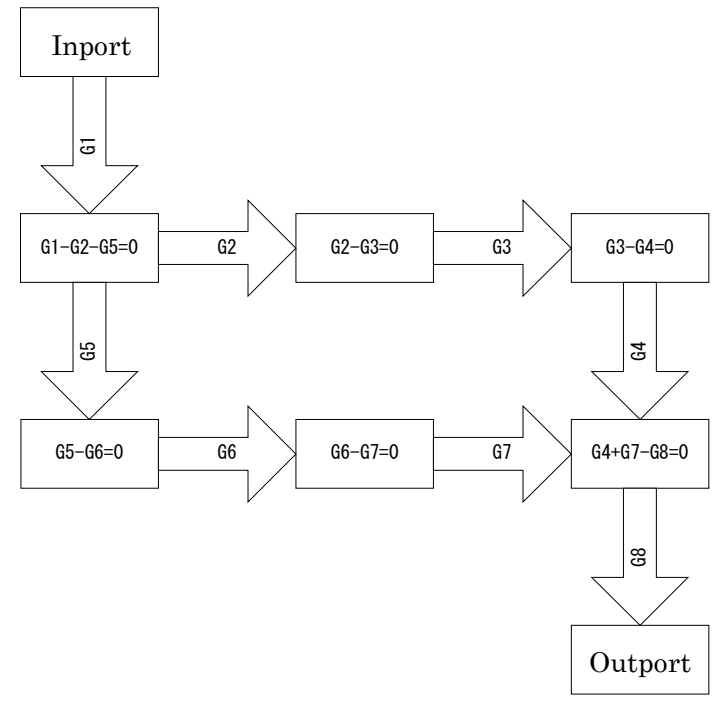

Fig. 2 Example system for the mass balance calculation

For instance, the following simultaneous Eq.(7) is used for the following system Fig.2.

$$
\left(\begin{array}{cccccccc}
1 & -1 & 0 & 0 & -1 & 0 & 0 & 0 \\
0 & 1 & -1 & 0 & 0 & 0 & 0 & 0 \\
0 & 0 & 1 & -1 & 0 & 0 & 0 & 0 \\
0 & 0 & 0 & 0 & 1 & -1 & 0 & 0 \\
0 & 0 & 0 & 0 & 0 & 1 & -1 & 0 \\
0 & 0 & 0 & 1 & 0 & 0 & 1 & -1 \\
\cdots & \ldots & \ldots & \ldots & \ldots & \ldots & \ldots & \ldots \\
\ldots & \ldots & \ldots & \ldots & \ldots & \ldots & \ldots & \ldots
\end{array}\right) \bullet\left(\begin{array}{c}
G_{1} \\
G_{2} \\
G_{3} \\
G_{4} \\
G_{5} \\
G_{6} \\
G_{7} \\
G_{8}
\end{array}\right)=\left(\begin{array}{c}
0 \\
0 \\
0 \\
0 \\
0 \\
\ldots \\
\ldots
\end{array}\right)
$$

However, it is not necessary to solve the simultaneous equations in the part of the relationship that has no loops. So, the values which are able to be uniquely determined are calculated first.

$$
G_{8}=G_{1}, G_{3}=G_{4}=G_{2}, G_{6}=G_{7}=G_{5}
$$

And then, only the equations associated with the remaining part are arranged in a matrix.

$$
\left(\begin{array}{ccc}
1 & -1 & -1 \\
\cdots & \ldots & \ldots \\
\ldots & \ldots & \ldots
\end{array}\right) \bullet\left(\begin{array}{l}
G_{1} \\
G_{2} \\
G_{5}
\end{array}\right)=\left(\begin{array}{c}
0 \\
\ldots \\
\ldots
\end{array}\right)
$$

This method makes the matrix smaller and easier to solve very huge matrix of entire power plant system. And we developed the program that can arrange the matrix like Eq.(9) quickly. 


\section{Checking the equations}

If the simultaneous equation includes the non-independent equation, it can not be solved. Therefore, it is necessary to check not only the number of equations and number of independent variables, but also that each equation is independent.

In order to correct the number of equations, the priority level is put on each equation. For instance, the conservation law has the highest priority, and the equation for pressure drop using default value has low priority. Then choose sequentially from the equation with a high priority.

For instance, in calculating the mass balance of Fig. 3, if $G_{2}$ and $G_{5}$ are set to different values, it can not be solved. In order to avoid such a situation, independent variable $x_{i}$ is prepared besides $G_{i}$.

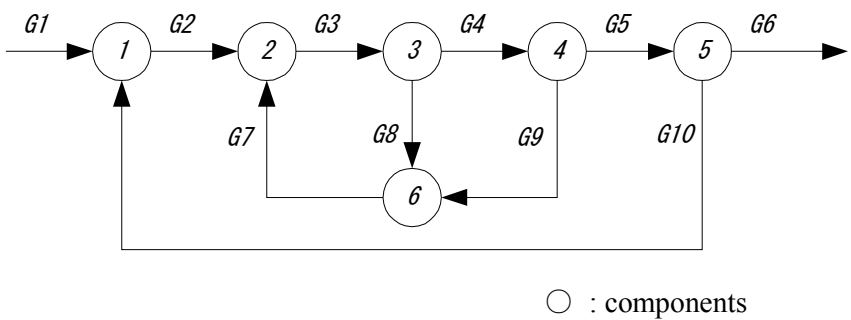

Fig. 3 Example system

Initially, the each $x_{i}$ corresponds to $G_{i}$

$\begin{array}{ll}G_{1}=x_{1} & G_{2}=x_{2} \\ G_{3}=x_{3} & G_{4}=x_{4} \\ G_{5}=x_{5} & G_{6}=x_{6} \\ G_{7}=x_{7} & G_{8}=x_{8} \\ G_{9}=x_{9} & G_{10}=x_{10}\end{array}$

Then the equation of each component is applied to reduce $x_{i} \mathrm{~s}$. At component 1 , the conservation law " $G_{1}+G_{10}=G_{2}$ " is used to eliminate the variable $x_{10}$. After the equation of the other component is sequentially applied, all $G_{i}$ is given by using only four independent variables $x_{1}$ to $x_{4}$.

$$
\begin{array}{ll}
G_{1}=x_{1} & G_{2}=x_{2} \\
G_{3}=x_{3} & G_{4}=x_{4} \\
G_{5}=x_{2} & G_{6}=x_{1} \\
G_{7}=x_{3}-x_{2} & G_{8}=x_{3}-x_{4} \\
G_{9}=x_{4}-x_{2} & G_{10}=x_{2}-x_{1}
\end{array}
$$

Thus, the number of the independent variables has been reduced to four, only by applying the conservation law. In this step, it is understood that the values of $G_{6}$ have to be the same as that of $G_{l}$.

Then, if the equation " $G_{2}=100$ " is set. The value of $x_{2}$ becomes " 100 " using the equation " $G_{2}$ $=x_{2}$ ", and then $x_{2}$ in the Eq.(11) are replaced by " 100 ".

$$
\begin{array}{llrl}
G_{1} & =x_{1} & & G_{2}=100 \\
G_{3} & =x_{3} & & G_{4}=x_{4} \\
G_{5} & =100 & & G_{6}=x_{1} \\
G_{7} & =x_{3}-100 & & G_{8}=x_{3}-x_{4} \\
G_{9} & =x_{4}-100 & & G_{10}=100-x_{1}
\end{array}
$$


Here, the value of $G_{5}$ becomes fixed value and it is clear that this is not able to be set to another value. Similarly, after the equations " $G_{3}=1000$ " and " $G_{1}=G_{10}$ " are applied, the following equations yield.

$$
\begin{aligned}
& G_{1}=50 \quad G_{2}=100 \\
& G_{3}=1000 \quad G_{4}=x_{4} \\
& G_{5}=100 \quad G_{6}=50 \\
& G_{7}=900 \quad G_{8}=1000-x_{4} \\
& G_{9}=x_{4}-100 \quad G_{10}=50
\end{aligned}
$$

Then, if the next equation is " $G_{I}=G_{6}$ ", ignore this equation and require next equation. And if the next equation is " $G_{2}=G_{6}$ ", send warning message and require another equation.

\section{Calculation technique of entire system}

In the calculation of the total system, $P, G$ and $H$ are calculated separately in following order.

1) Calculate the pressure balance.

2) Calculate the mass balance.

3) (if needed) Calculate the pressure from flow rate, and return to 1).

4) Calculate the enthalpy balance.

5) (if needed) Calculate the pressure from enthalpy, and return to 1).

6) (if needed) Calculate the mass flow rate from enthalpy, and return to 1).

In each step, some methods mentioned above are combined and used, according to the system configuration and calculation conditions. The step 3), 5) and 6) enable us to calculate the heat and mass balance of entire system under various conditions.

This is a very useful feature of our software; it is possible to calculate the mass flow rate from given temperature and the pressure drop from given mass flow rate, etc. For, the mass flow rate of extraction steam must be calculated from the given inlet and outlet temperature of working fluids of heat exchanger, or the initial pressure must be determined by the given mass flow rate of main steam.

\section{Drawing the system}

Figure 4 shows the execution window of this software and No.2 low pressure feed water heater (Heater2) of nuclear power plant is shown here. The procedure for drawing the system is explained with this simple example.

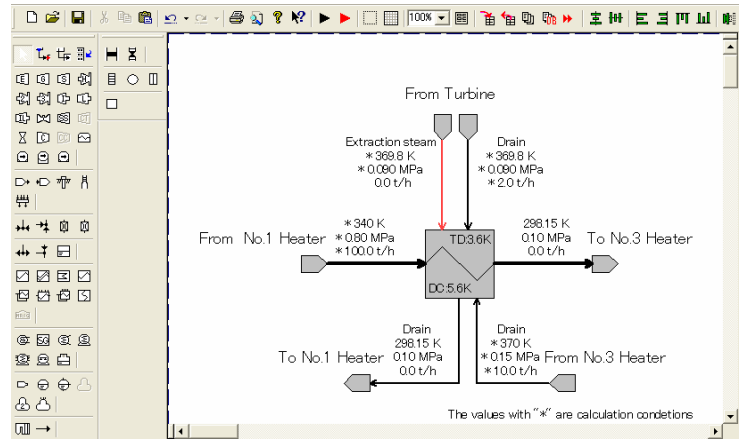

Fig. 4 Example system

No.2 low pressure feed water heater of nuclear power plant 
First, under the drawing mode, select required components, which are three inlet port models, two outlet port models and a crossflow heat exchanger model, from the palette on the extreme left of window, and arrange them on the canvas. Next, under the piping mode, connect the components with pipes as shown in the illustration. Then input the calculation conditions. Here, following conditions are set.
1) Condensate flow rate
$: 100 \mathrm{t} / \mathrm{h}$
2) Feed water inlet pressure
$: 0.8 \mathrm{MPa}$
3) Feed water inlet temperature
: $340 \mathrm{~K}$
4) Extraction steam pressure
: $0.09 \mathrm{MPa}$
5) Quality of extraction steam
$: 0.95$
6) Turbine drain flow rate
$: 2 \mathrm{t} / \mathrm{h}$
7) Terminal temperature difference (TD) of Heater2
$: 3.6 \mathrm{~K}$
8) Initial temperature difference (DC) of Heater2
$: 5.6 \mathrm{~K}$

But the calculation conditions are still insufficient, undermentioned default conditions are used in the calculation.

1) Heater2 pressure drop of condensate flow

$: 0 \%$

2) Heater 2 pressure drop of extraction flow

$: 0 \%$

Figures 5 and 6 show the calculation results. After calculation, the values such as extraction steam flow rate, heat exchanging performance, etc. are computed. Moreover, if extraction steam flow rate was set as calculation condition, the TD; that is the feed water outlet temperature of Heater2, can be obtained after calculation. And we can also calculate the fluid temperatures around Heater2 by coefficient of heat transmission of Heater2.

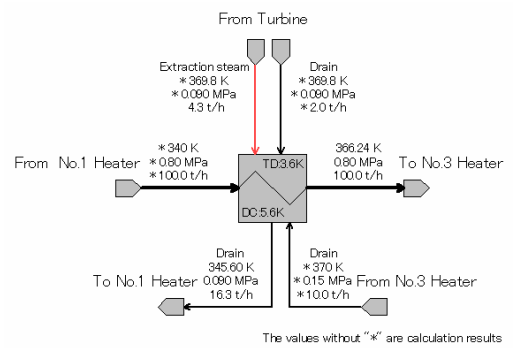

Fig. 5 Calculation results of the system; Heater2

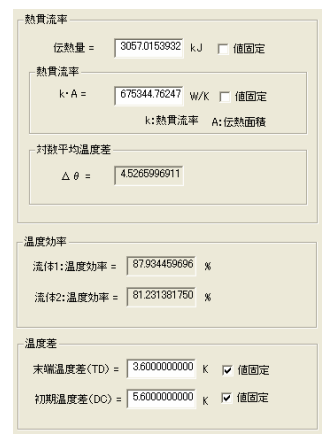

Fig. 6 Heat exchanging performance of Heater2 


\section{Analysis of nuclear power plant system}

Figure 7 shows the essential component models required to calculate the heat and mass balance of nuclear power plant system. We have developed more than twenty component models, and the IAPWS IF-97 steam tables ${ }^{[4]}$ and subroutines to calculate the thermodynamic properties of main gas elements ${ }^{[5]}$ are included in the software.

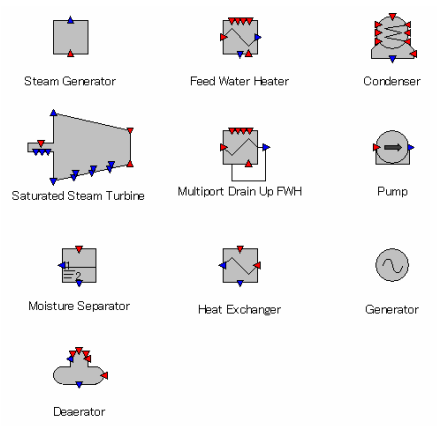

Fig. 7 main components to analyze nuclear power plant included in the developed software

Figures $8 \sim 11$ show the heat and mass balance diagrams of main steam system of nonexistent nuclear power plant with different heat recovering system of steam generator blowdown water. Fig. 8 shows the system that exhausts the blowdown water into the condenser directly (system1). Fig.9 shows the system in which the blowdown water is utilized as thermal source to heat the low pressure feed water (system2). Figs.10 and 11 show the systems in which the blowdown water is recovered into the deaerator and the high pressure feed water heater (Heater5), respectively (system3 and 4).

Without this software, we must make another calculation program for each system. However, using this software, it is easy to make the calculation program of another system from the other one. The only step that needs to be performed is cut \& paste the required components of heat and mass balance diagram, reconnect them on the canvas like a drawing application, and set the calculation conditions.

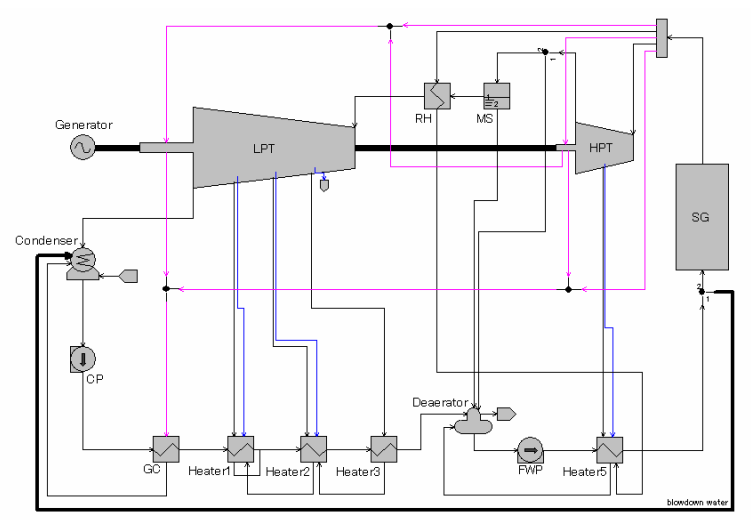

Fig. 8 Heat and mass balance diagram of system1 


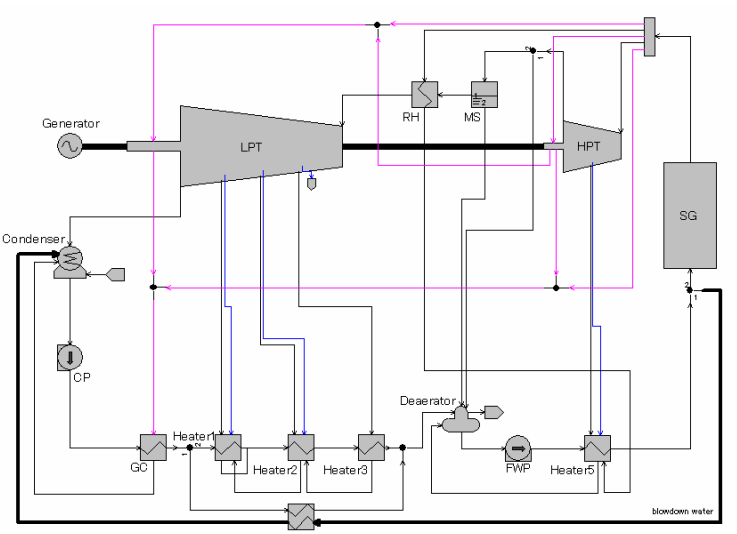

Fig. 9 Heat and mass balance diagram of system2

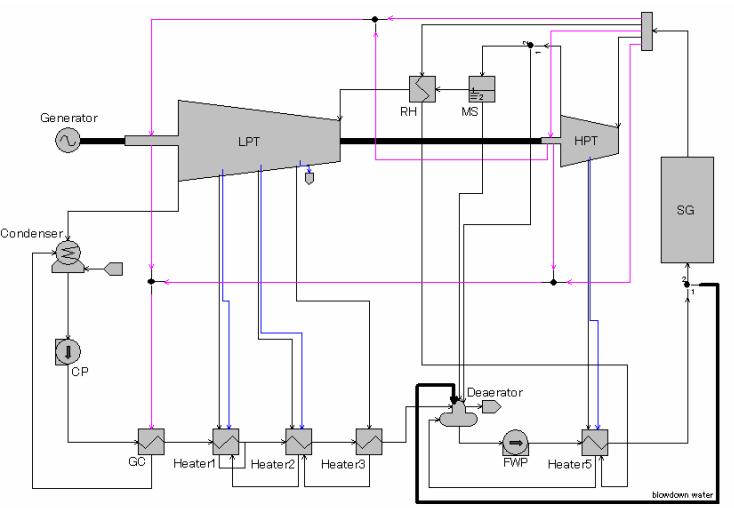

Fig. 10 Heat and mass balance diagram of system 3

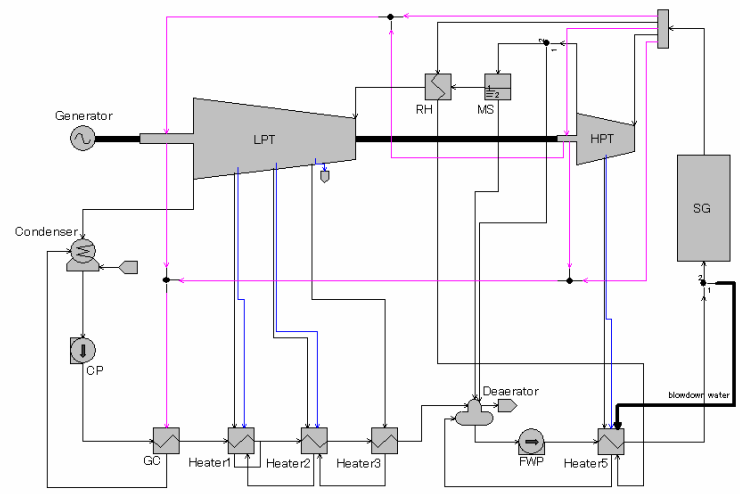

Fig. 11 Heat and mass balance diagram of system 4

We weighed the calculation results of each system under the following assumptions:

1) Flow rate of blowdown water is $1 \%$ of that of main feed water

2) Constant heat output

3) Pressure of turbine extractions and flow rate of turbine drains are related to inlet flow rate of each turbine

4) TD and DC of each heat exchanger are fixed 
Figure 12 shows the increase rate per the gross power output of system1. The blowdown water is recovered to each part of system, it is not necessary to heat main feed water utilizing extraction steam. The decrease of extraction steam flow rate from each turbine port then means that the main steam flow rate increases, thereby the power output of each turbine increases. Besides, we see from these results that recovering the blowdown water to heat exchanger which operates at the temperature as same level as one of blowdown water leads to the most improvement of power output of the system. Thus, this software enables to draw the heat and mass balance diagrams of various systems and analyze the static characteristic under a variety of calculation condition. Moreover, using this software, we can simulate the effects of changing the vacuum to total plant performance and so on.

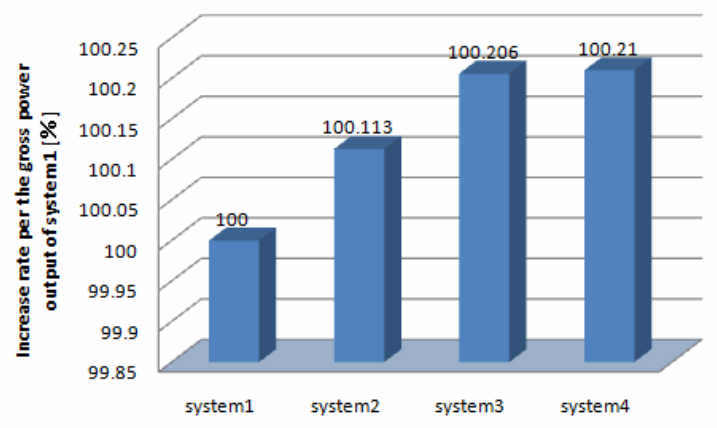

Fig. 12 Increase rate per gross power output of system1

\section{Conclusion}

We have developed the general-purpose software by which static thermal characteristic of the power generation system is analyzed easily. This software is designed with a new calculating method which has the flexibility for setting the calculation condition and for building new system component models of power plant into the software, and the ability to calculate faster. It proved to be clear that this method is applicable to analyze the performance of many types of power generation systems under various calculation conditions using a personal computer. This software could be utilized for planning of the plant maintenance strategy and its operation.

In the future, we are going to develop and add more component models and thermodynamic properties databases, and reinforce the function of this software. At the same time, we will keep searching for the more efficient system with this software.

\section{References}

[1] E. Koda, "Study on High Performance CAES Power Generation System Applying the Advanced Combined Cycle Technology", Proceedings of International Gas Turbine Congress, Kobe, Japan(1999)

[2] T. Takahashi, Y. Nakao, E. Koda, "Analysis and evaluation about Advanced humid air turbine system”, International Conference on Power Engineering-2007, Hangzhou, China(2007)

[3]Y. Nakada, "Development of Thermodynamics Performance Analysis Simulator for Geothermal Power Plant and the Evaluation of the Plant Operational Characteristics", First European Geothermal Review - Geothermal Energy for Power Production, Mainz, Germany(2007)

[4] Patent number:3857840 (patented in Japan) 